\title{
Résumé de la bourse Tonipharm
}

C Springer-Verlag France 2010

6

\author{
Résumé de la bourse Tonipharm \\ H. Damon \\ Hôpital Édouard-Herriot, place d'Arsonval, \\ F-69437 Lyon cedex, France
}

\section{ORALIA - IRMf \\ Étude par IRM fonctionnelle de l'effet \\ de la neuromodulation des racines sacrées sur le cortex cérébral chez des patients souffrant d'incontinence fécale sévère \\ H. Damon ${ }^{1}$, C. Scheiber ${ }^{2}$, X. Barth ${ }^{3}$, F. Mion ${ }^{1}$ ${ }^{1}$ Service d'exploration fonctionnelle digestive, hôpital Édouard-Herriot, CHU de Lyon, France \\ ${ }^{2}$ Service de médecine nucléaire, groupement hospitalier Est, \\ CHU de Lyon, France \\ ${ }^{3}$ Service de chirurgie digestive, hôpital Édouard-Herriot, CHU de Lyon, France}

Objectifs : L'objectif principal de cette étude est de décrire les modifications de la cartographie des zones corticales activées, établie par IRM fonctionnelle (IRMf), lors de la NMS chez des patients souffrant d'incontinence fécale sévère. L'objectif secondaire est de décrire la cartographie des zones corticales activées, établie par IRMf, chez des patients souffrant d'incontinence fécale sévère.

Hypothèse : L'hypothèse de cette étude est que la NMS stimulerait certaines zones du cortex cérébral.
Type d'étude : Il s'agit d'une étude descriptive de physiopathologie, unicentrique.

Critères d'inclusion : Cette étude s'adresse aux patients, de 18 à 75 ans, devant bénéficier d'un test de NMS pour incontinence fécale sévère (score de Wexner $\geq 10$ ).

Facteur étudié : Le facteur étudié sera la NMS.

Critère de jugement : Le critère de jugement sera l'identification des voxels (voxel = élément de volume du cerveau en imagerie) activés lors de la NMS sur la cartographie établie par IRMf.

Déroulement de l'étude : Un bilan initial, correspondant à celui d'une prise en charge habituelle dans le but de réaliser un test de NMS, permet de vérifier les critères d'inclusion et de proposer au patient de participer à l'étude. Deux examens par IRMf seront réalisés : le jour, ou le lendemain, de la mise en place de l'électrode test et du boîtier provisoire de NMS, puis au cours de la période de test de NMS, 15 jours après la pose.

En fin de période de test de NMS, la décision d'implantation définitive sera prise. Cette décision ne sera en aucun cas prise en fonction des résultats des examens d'IRM. Pour les patients ayant bénéficié d'une implantation définitive, une visite est programmée au sixième mois après l'implantation définitive.

La période d'inclusion sera de 12 mois. La durée totale de l'étude sera de deux ans.

Nombre de sujets nécessaires : 15 patients pourront être recrutés sur une période de 12 mois.

Analyse : Lors des deux examens, un logiciel approprié permettra d'identifier les différences d'activation corticale entre la NMS en position arrêt ou en position marche. 